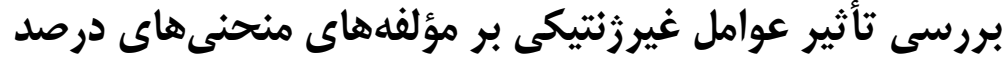

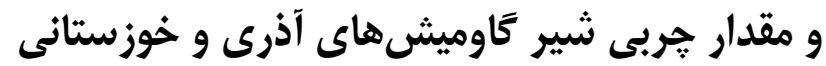

\author{
كريم حسن يور '، سيد عباس رافتَّ، آرش جوانمردّو داوود كيانزادء
}

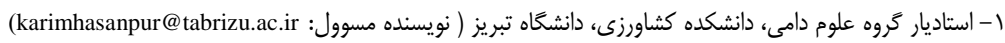

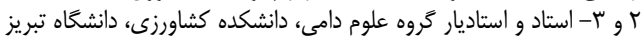

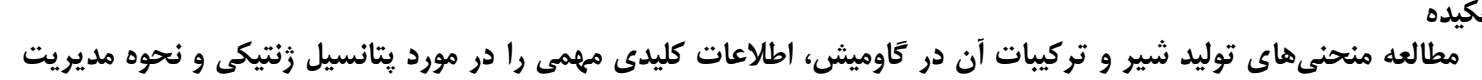

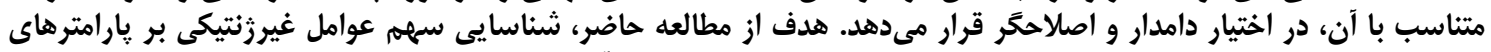

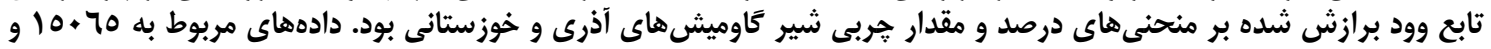

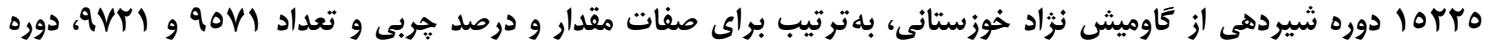

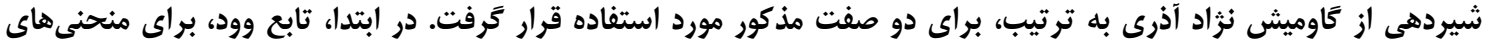

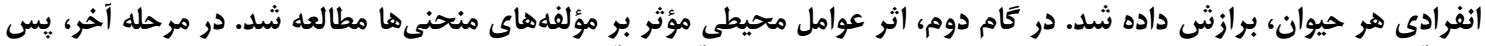

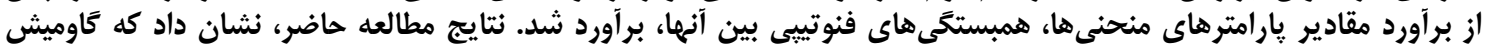

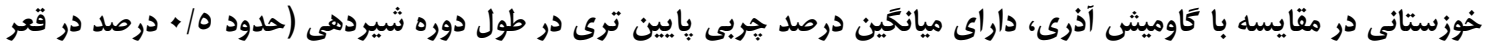

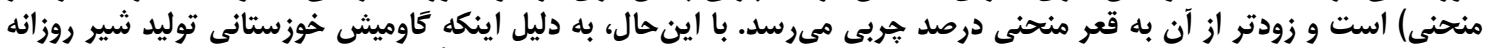

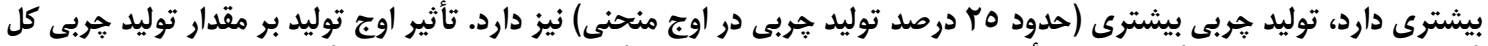

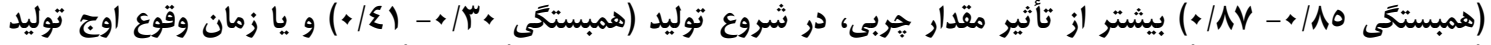

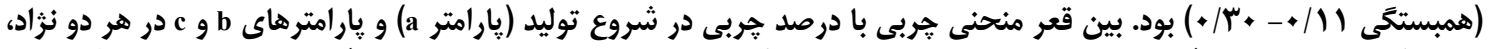

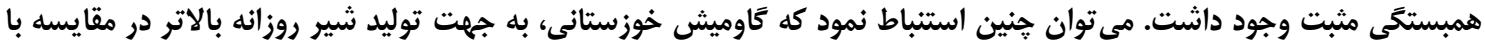

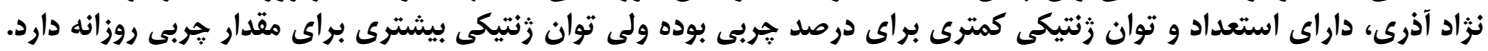

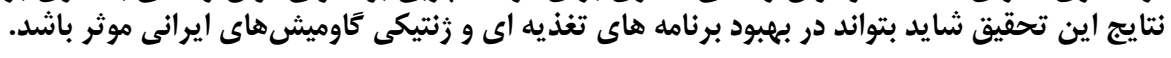

وازههاى كليدى: كاوميش، منحنى خربى شير، تابع وود، اوج توليد، همبستكى

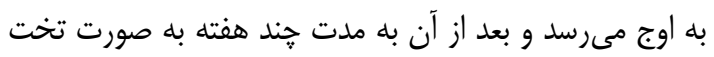

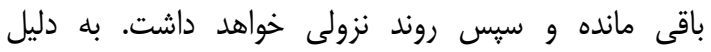

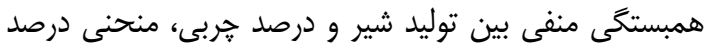

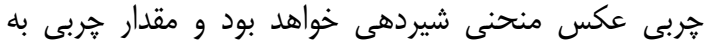

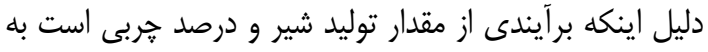

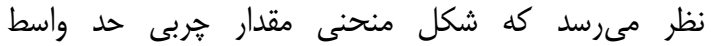

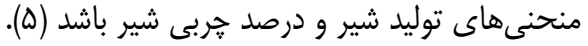

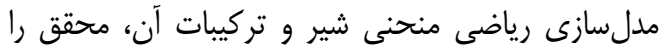

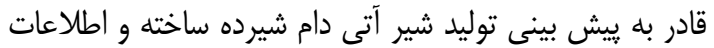

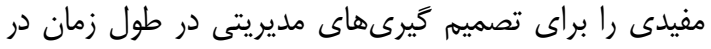

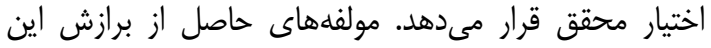

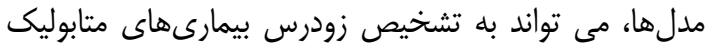

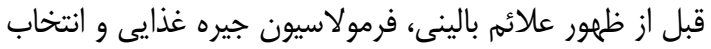

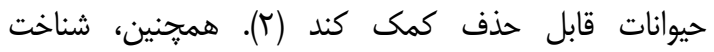

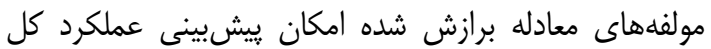

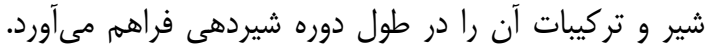

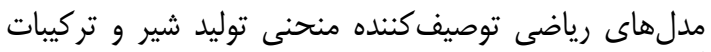

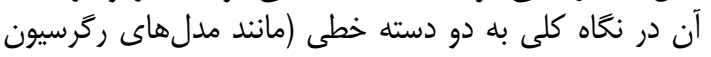

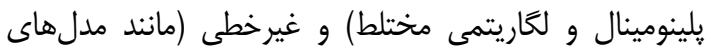

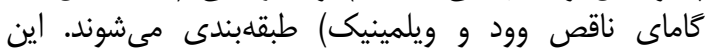

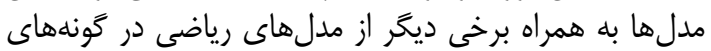

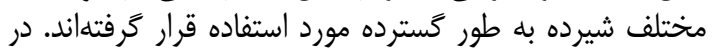

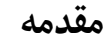

كاوميشهاى بومى ايران، از جمله ذخاير زنتيكى مهام

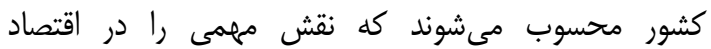

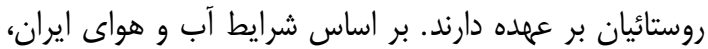

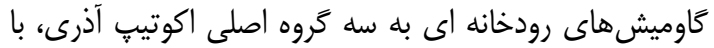

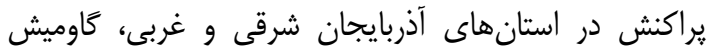

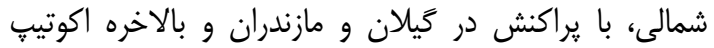

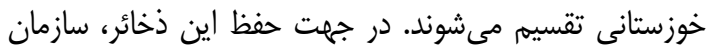

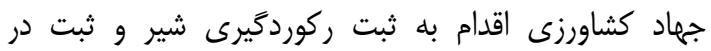

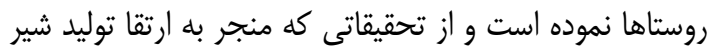

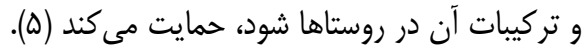

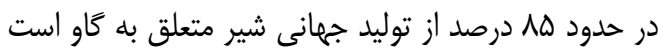

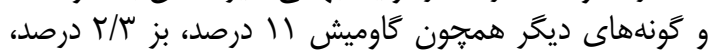

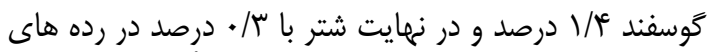

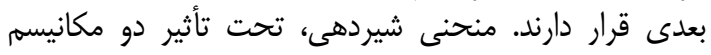

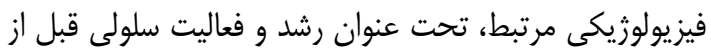

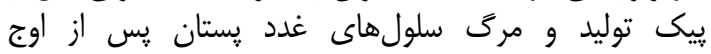

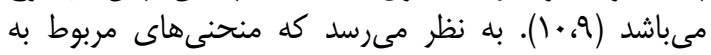

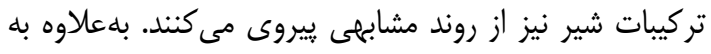

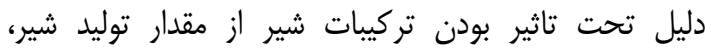

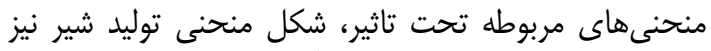

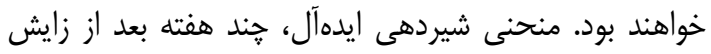


آنها با نزاد آذرى، در گروه موسوم به نزاد آذرى قرار داده

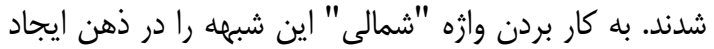

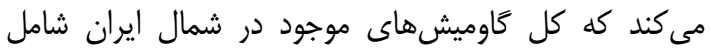

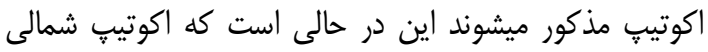

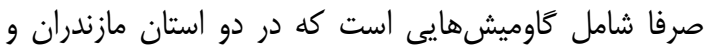

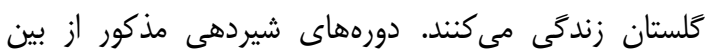

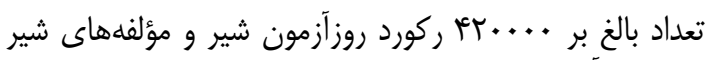

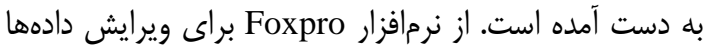

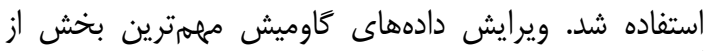

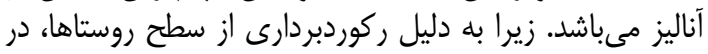

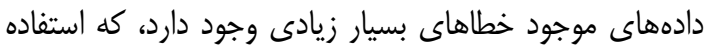

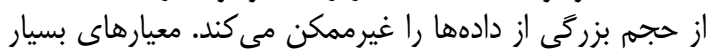

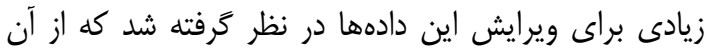
جمله ميتوان به موارد زير اشاره كرداي

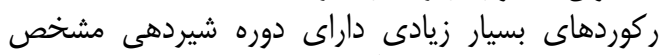

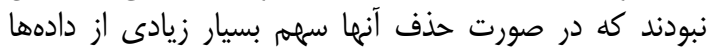

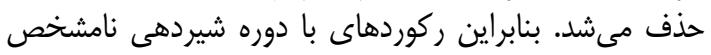

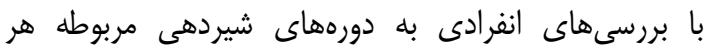

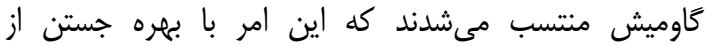

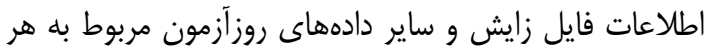

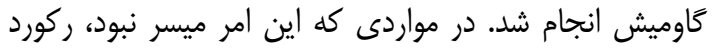

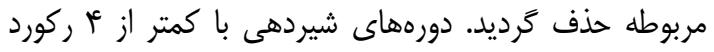

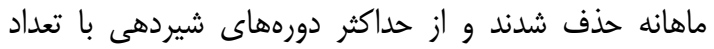

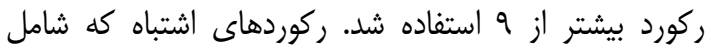

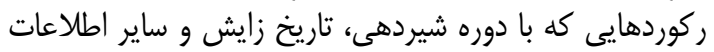

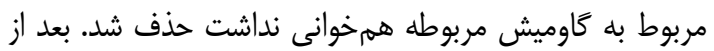

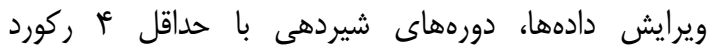

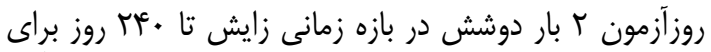

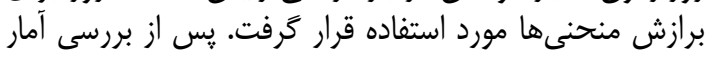

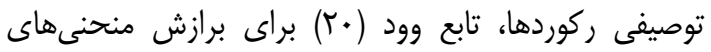

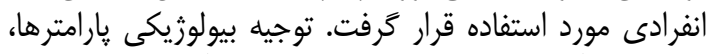

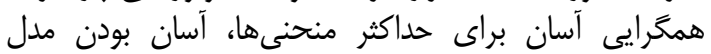

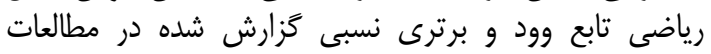

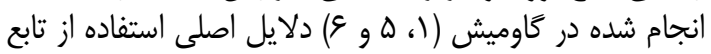

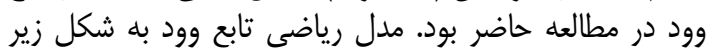

$$
y_{t}=a t^{b} e^{-c t}
$$

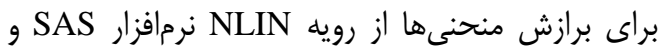

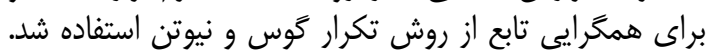

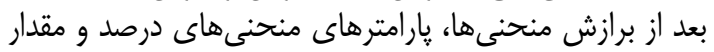

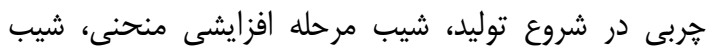

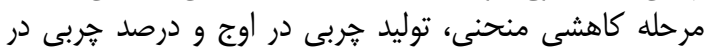

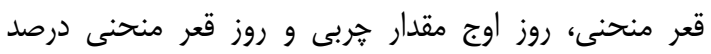

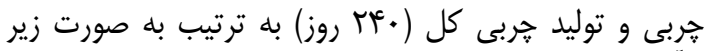

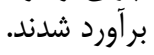

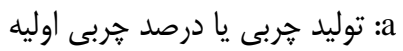

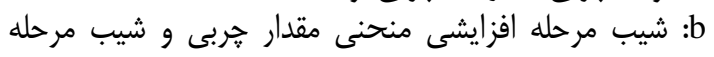
كاهشى منحنى درصد خربى افيى منحنى
نزادهاى مختلف كاوميش نيز از اين توابع براى برازش

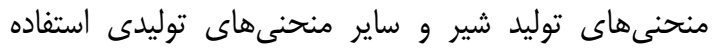

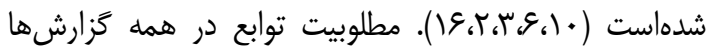

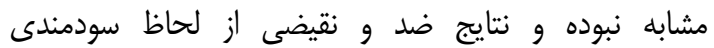

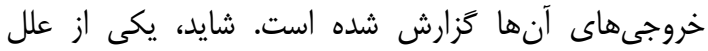

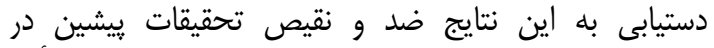

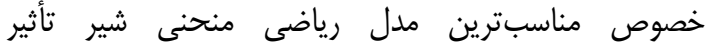

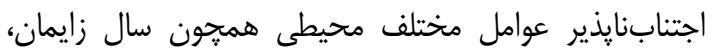

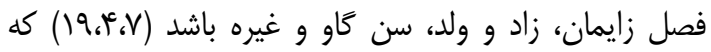

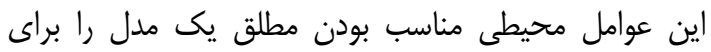

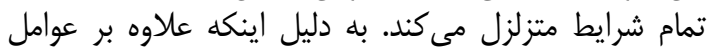

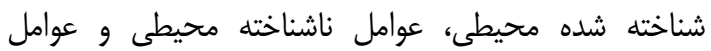

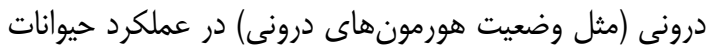

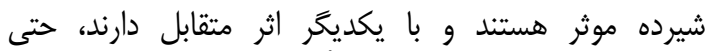

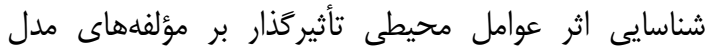

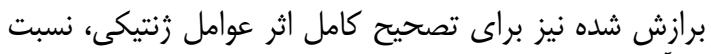

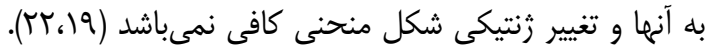

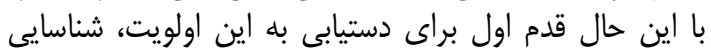

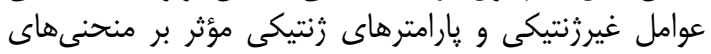

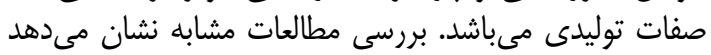

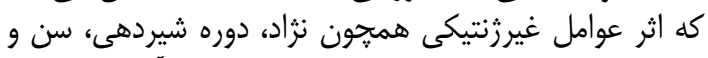

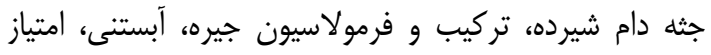

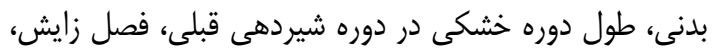

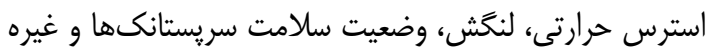

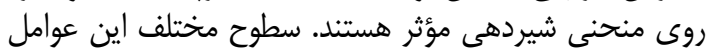

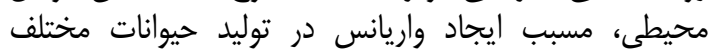

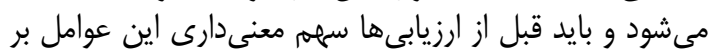

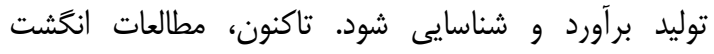

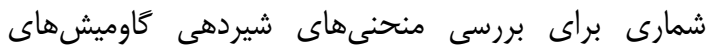

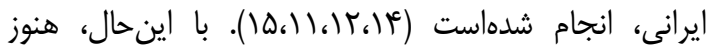

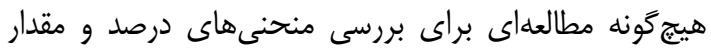
קربى شير كاوميشهاى كشور انجام نشده است. لذايكائ هدف از

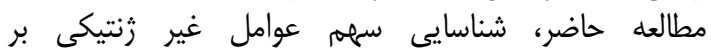

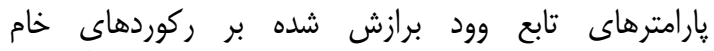

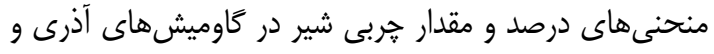

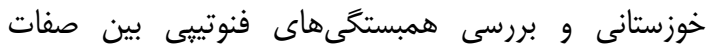
مختلف منحنى هاى مذكور بود.

\section{مواد و روشها ماريا}

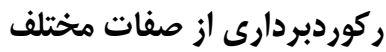

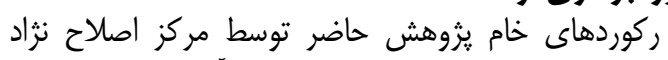

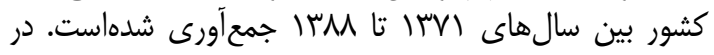

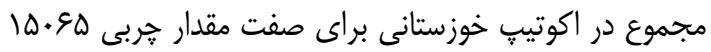

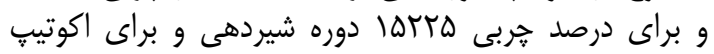

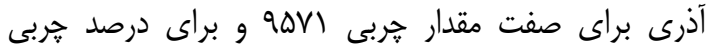

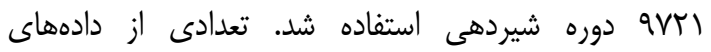
كاوميشهاى استانهاى شمالى كشور نيز، در بانكى اطلاعاتى موجود بود كه به دليل شباهت نسبى مقدار و الكَّى نور توليدى 
v روستا: اثر روستا جايكزين اثر كله شد. از آنجايى كه

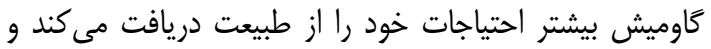

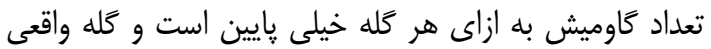

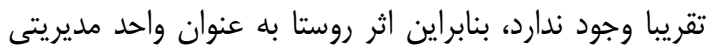

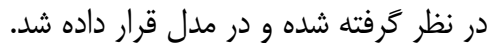
管

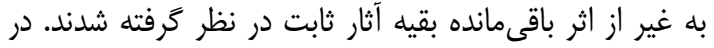

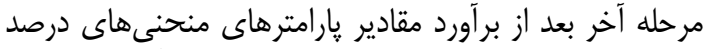

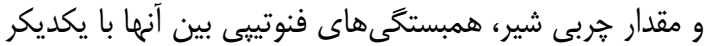

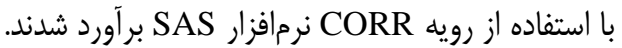

\section{نتايج و بحث مؤر آمار توصيفى مؤلفههاى مدل وود براى خصوصيات جربى} تنابع وود (كاماى ناقص) بسيارى از منحنىها را به راحي راحتى

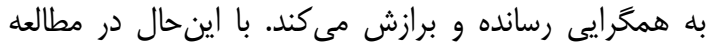

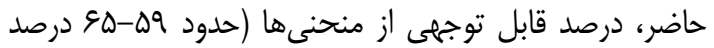

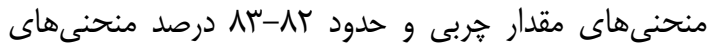

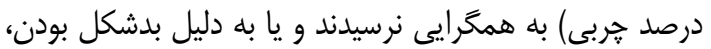

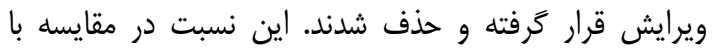
ميزان منحنىهاى بدشكل توليد

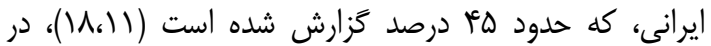

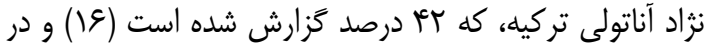

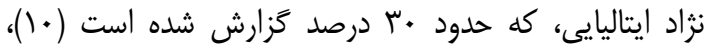

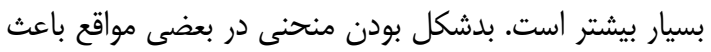

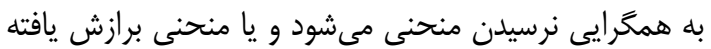

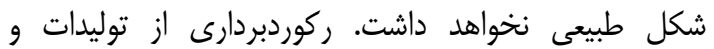

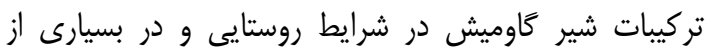

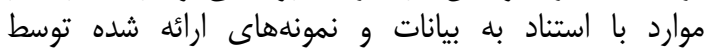

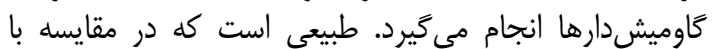

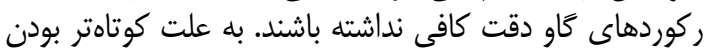

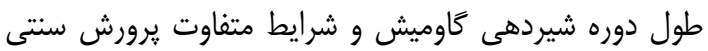

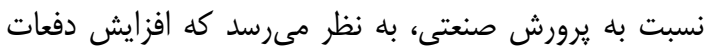

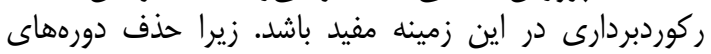

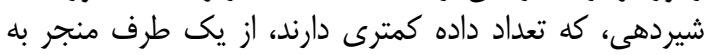

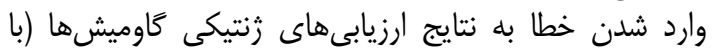

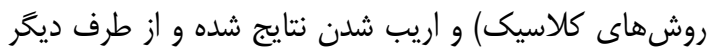

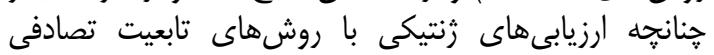

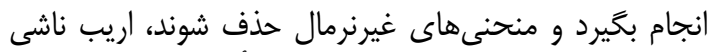

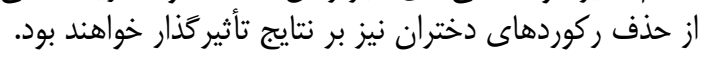

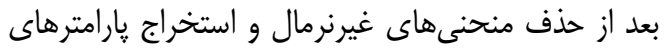

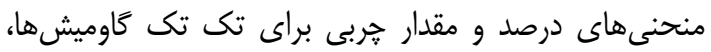

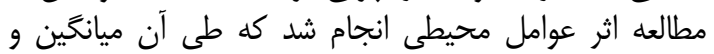

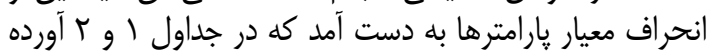

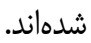

c c: شيب مرحله كاهشى منحنى مقدار קربى و شيب مرحله

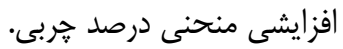

اوج مقدار هربى يا كمترين درصد جربى (قعر منحنى).

$$
P_{y}=a(b / c)^{b} e^{-b}
$$
روز اوج توليد جربى يا كمترين درصد جربى

$P_{d}=b / c$

توليد קربى • • (ז) روز

$$
F a t Y=a \int_{1}^{240} t^{b} \exp ^{-c t} d t
$$

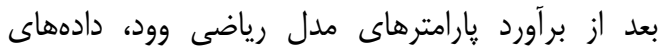

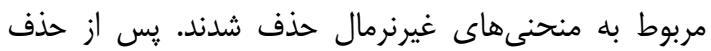

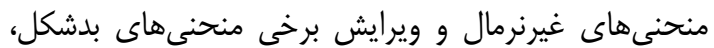

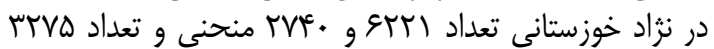

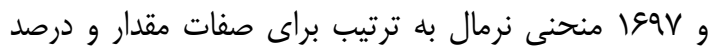

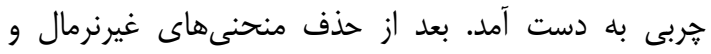

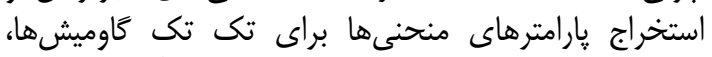

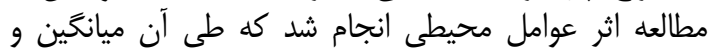

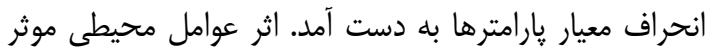

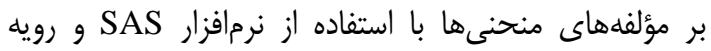

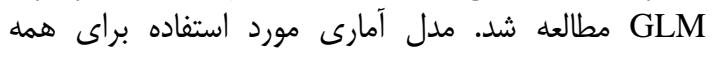
صفات به قرار زير بود: $y_{i j k m n}=\mu+b y_{i}+c y_{j}+a g e_{k}+c_{l}+c s_{m}+v_{n}+e_{i j k l m n}$ كه در آن: - - آن קربي : مشاهدات مربوط به هر كدام از صفات منحنى مقدار جربى و درصد هربى شير:

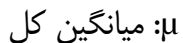
by cy

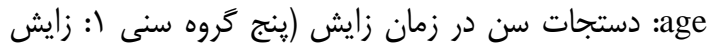

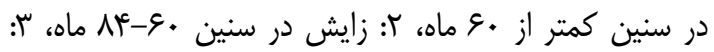

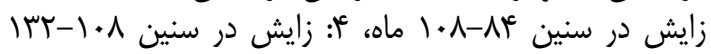

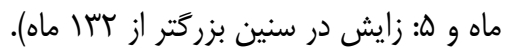

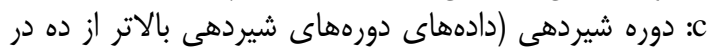

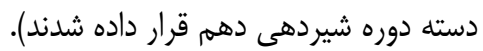

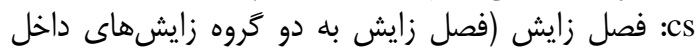
فصلى (زايشهايى كه در فرايش فصول تابستان و وإياييز اتفاق

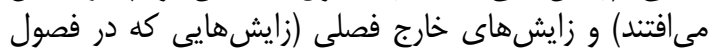

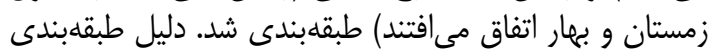

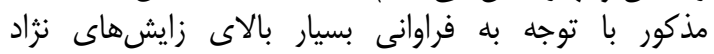

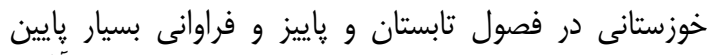

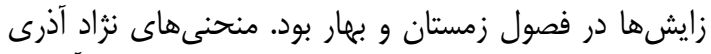

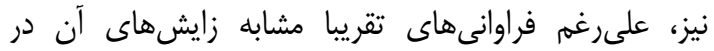
فصول مختلف سال، در دو فصل طبقابندى شدندان. 
جدول ا - آمار توصيفى هارامترهاى منحنى درصد هربى شير نزادهاى كَاوميش خوزستانى و آذرى Table 1. Descriptive statistics of milk fat percentage curve parameters for Azari and Khuzestani buffalo breeds

\begin{tabular}{|c|c|c|c|c|c|c|c|}
\hline $\mathrm{p}_{\mathrm{d}}$ & $\mathrm{p}_{\mathrm{y}}$ & $\mathrm{c}$ & $\mathrm{b}$ & $\mathrm{a}$ & تعداد & & \\
\hline GT & $\Delta /{ }^{\circ} \varphi$ & $-\cdot \cdot \cdot \cdot \varphi^{c}$ &.$- \cdot 199$ & 1.140 & \multirow{2}{*}{$T V F^{e}}$. & ميانگين & \multirow{2}{*}{ خوزستانى } \\
\hline$r \cdot / \Delta$. & . & . &.$/ 1 \mathrm{~V}$ & $r / \Lambda 1$ & & انحراف معيار & \\
\hline SD/IV & $0 / Q \varphi$ & r.|.|- &.$- / 189$ & $1 . / 95$ & \multirow{2}{*}{1998} & ميانخين & \multirow{2}{*}{ آذرى } \\
\hline MI/TF & פשו/1 & . /.r &.$/ 11$. & $r / v e$ & & انحراف معيار & \\
\hline
\end{tabular}

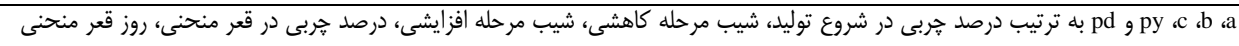

جدول r- آمار توصيفى پارامترهاى منحنى مقدار تربى شير نزادهاى گاوميش خوزستانى و آذرى Table 2. Descriptive statistics of milk fat content curve parameters in Azari and Khuzestan buffalo breeds

\begin{tabular}{|c|c|c|c|c|c|c|c|c|}
\hline FatY & $\mathrm{p}_{\mathrm{d}}$ & $\mathrm{p}_{\mathrm{y}}$ & $\mathrm{c}$ & $\mathrm{b}$ & a & تعداد & & \\
\hline IFT/V & $\Lambda \varepsilon / \mu$ & $\cdot / V \Delta$ & $.1 \cdot .9$ & $\cdot / V \Delta \Lambda$ & $\cdot / 4 \cdot 1$ & \multirow{2}{*}{ STrI } & ميانگين & \multirow{2}{*}{ خوزستانى } \\
\hline.$/ 4 q$ & MTE/Tr & G & $.1 .+9$ & $\cdot / \mathrm{V}$ & ./TTA & & انحراف معيار & \\
\hline$m(F)$ & $r \omega / r q$ & $\cdot / T \cdot \Delta$ & $\cdot 1 \cdot+1$ &.$/ 94$ &.$/ 19 V$ & TrVD & انحراف معيار & آذرى \\
\hline
\end{tabular}

از حصول يارامترهاى منحنىهاى مقدار و درصد جربى، از آنها

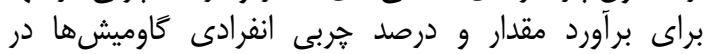

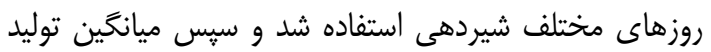

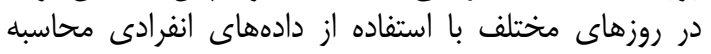

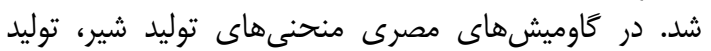

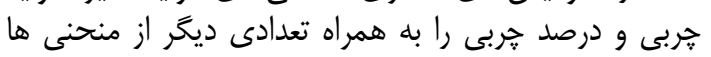

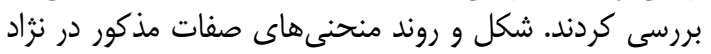

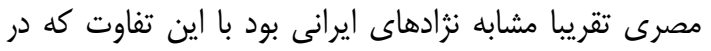

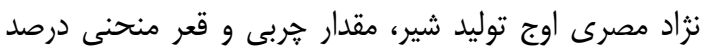

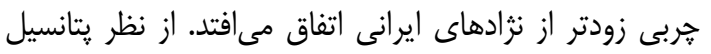

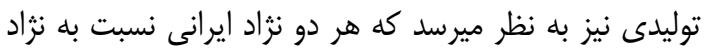

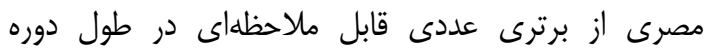

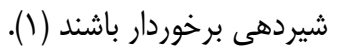

نتايج اين :زئهش نشان داد كه، كاوميشهاى خوزستانى

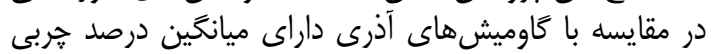

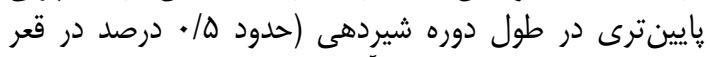

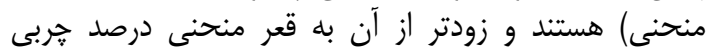

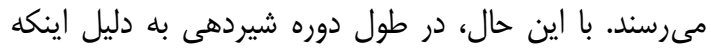

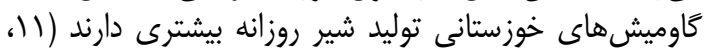

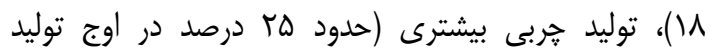

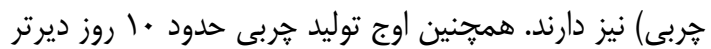

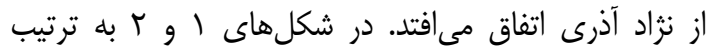

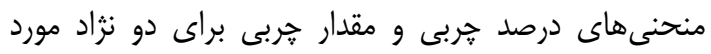

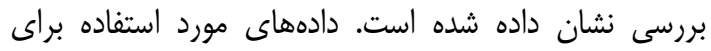

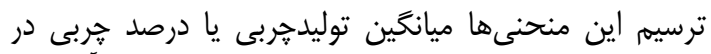

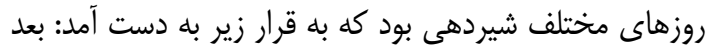

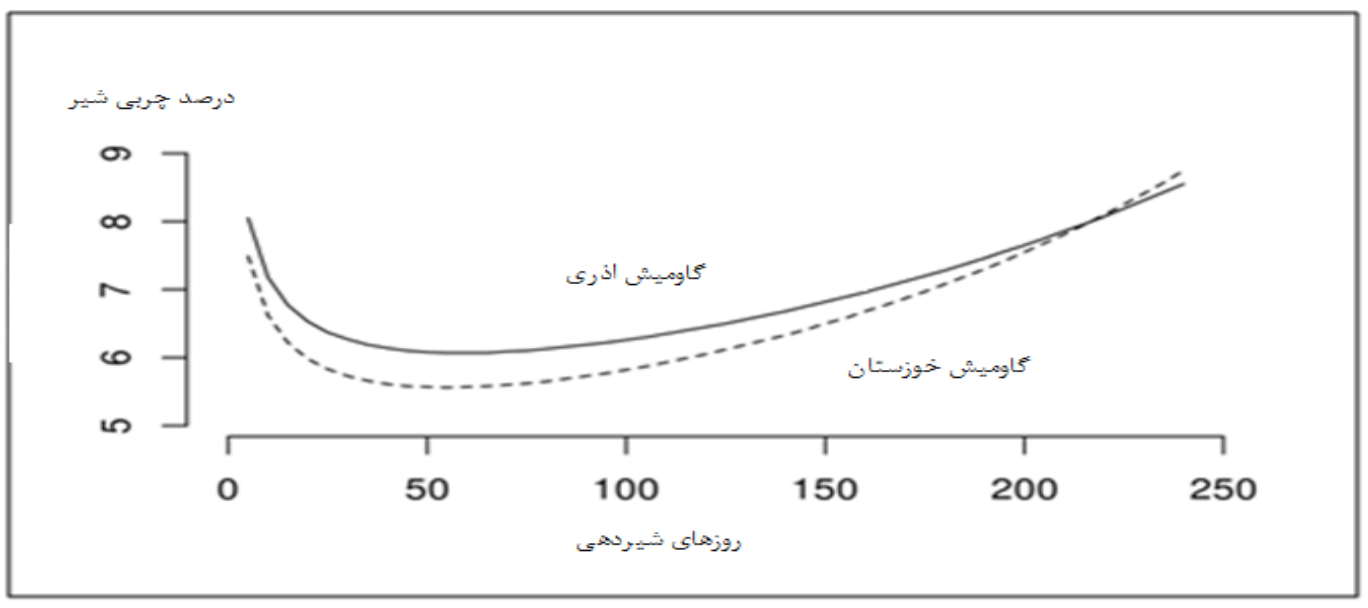

نمودار ا- منحنى توصيف كننده تغييرات ميانكين درصد جربى در كل دوره شيردهى كاوميشهاى آذرى و خوزستانى Figure 1. Descriptive curve for variation of milk fat curve parameters in Azari and Khuzestani buffalo breeds 


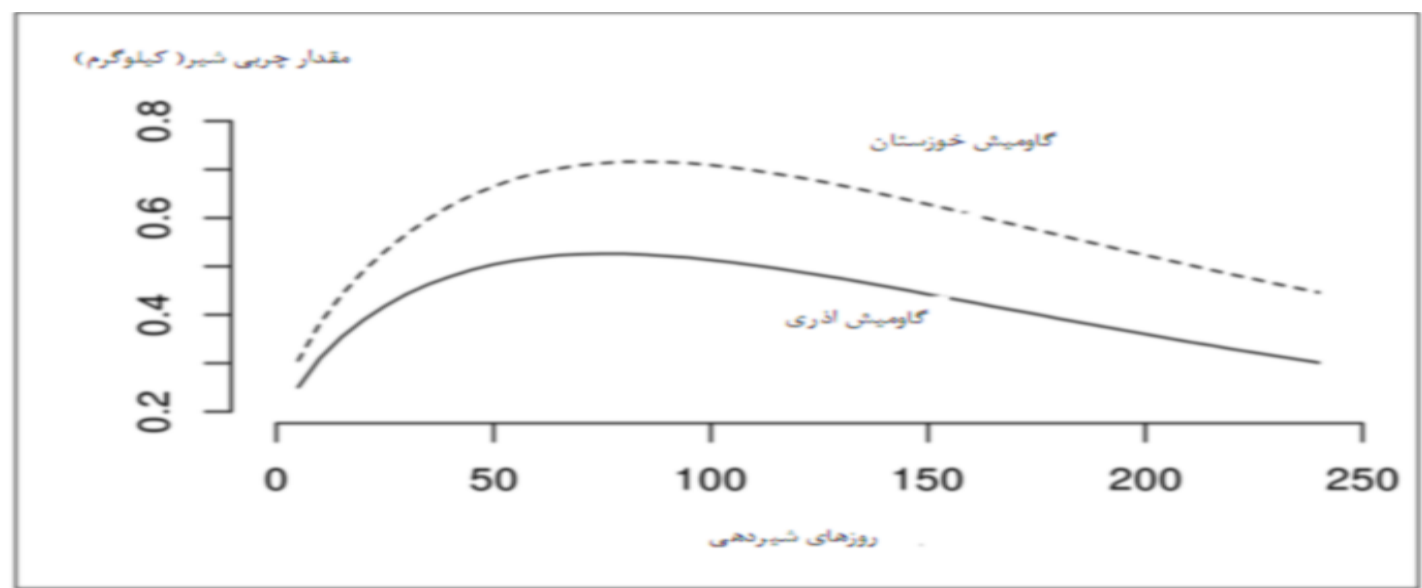

نمودار r- منحنى توصيف كننده تغييرات ميانخين مقدار جربى در كل دوره شيردهى كاوميش هاى آذرى و خوزستانى Figure 2. Descriptive curve for variation of milk fat curve parameters in Azari and Khuzestani buffalo breed

جربى آورده شدهاند. از ذكر جزئيات و ميانخين حداقل مربعات

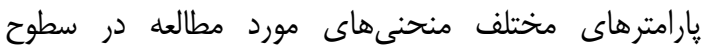
مختلف فاكتورها خوددارى مى شود.

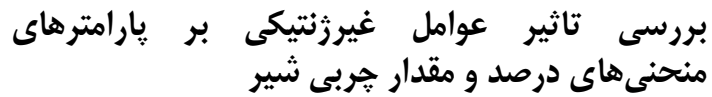

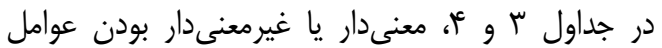

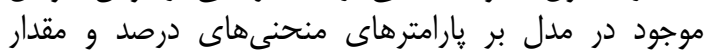

Table 3. Statistical of non-genetic effect on fat percentage curve parameters

جدول ب- تأثير آمارى عوامل غير زنتيكى بر پارامترهاى منحنى درصد جربى

\begin{tabular}{|c|c|c|c|c|c|c|c|c|c|c|c|c|}
\hline \multicolumn{6}{|c|}{ نثزاد آذرى } & \multicolumn{6}{|c|}{ نزاد خوزستانى } & \\
\hline $\mathrm{p}_{\mathrm{d}}$ & $\mathrm{p}_{\mathrm{y}}$ & $\mathrm{c}$ & $\mathrm{b}$ & $\mathrm{a}$ & $\mathrm{Df}$ & $\mathrm{p}_{\mathrm{d}}$ & $\mathrm{p}_{\mathrm{y}}$ & $\mathrm{c}$ & $\mathrm{b}$ & $\mathrm{a}$ & df & \\
\hline * & ns & ns & ns & ns & r & ** & ns & ns & ns & ns & TV & سال تولد \\
\hline ** & $* *$ & ** & * & ns & is & ** & $* *$ & ** & $* *$ & ** & 19 & سال زايش \\
\hline ns & $*$ & $\mathrm{~ns}$ & ns & ns & f & $*$ & $\mathrm{~ns}$ & ns & ns & ns & r & دستجات سنى \\
\hline ** & $* *$ & ** & ** & ns & r. & $* *$ & $* *$ & ** & ** & ** & is & ستا \\
\hline ** & $\mathrm{ns}$ & ns & * & * & 9 & ns & * & ns & ns & ns & 9 & دوره شيردهى \\
\hline ns & $* *$ & ns & ns & ns & 1 & $\mathrm{~ns}$ & ns & ns & $* *$ & $\mathrm{~ns}$ & 1 & فصل زايش \\
\hline
\end{tabular}

جدول عأ تأثير آمارى عوامل غير زنتيكى بر يارامترهاى منحنى مقدار خربى Table 4. Statistical of non-genetic effect on fat content curve parameters

\begin{tabular}{|c|c|c|c|c|c|c|c|c|c|c|c|c|c|c|}
\hline \multicolumn{7}{|c|}{ نزراد آذرى } & \multicolumn{7}{|c|}{ نزاد خوزستانى } & \\
\hline FatY & $\mathrm{p}_{\mathrm{d}}$ & $\mathrm{p}_{\mathrm{y}}$ & $\mathrm{c}$ & $\mathrm{b}$ & $\mathrm{a}$ & df & FatY & $\mathrm{p}_{\mathrm{d}}$ & $\mathrm{p}_{\mathrm{y}}$ & $\mathrm{c}$ & $\mathrm{b}$ & $\mathrm{a}$ & df & \\
\hline ns & ns & $* *$ & $\mathrm{~ns}$ & ns & $\mathrm{ns}$ & זr & $* *$ & $* *$ & $* *$ & ns & ns & $*$ & tr & سال تولد \\
\hline ** & ** & ** & ** & ** & ** & $1 f$ & $* *$ & $* *$ & $* *$ & ** & $* *$ & ** & 19 & سال زايش \\
\hline $\mathrm{ns}$ & ns & ns & ns & ns & ns & f & $* *$ & $*$ & $* *$ & ns & ns & ns & f & دستجات سنى \\
\hline$* *$ & ** & $* *$ & $* *$ & $* *$ & $* *$ & rV & $* *$ & $*$ & $* *$ & ** & $* *$ & ** & pe & روستا \\
\hline$* *$ & $\mathrm{~ns}$ & $* *$ & ns & ns & ns & 9 & $* *$ & ns & $* *$ & $*$ & ns & ns & 9 & دوره شيردهى \\
\hline ns & ns & ns & $\mathrm{ns}$ & ns & ns & 1 & ns & $* *$ & ns & $* *$ & ns & ns & 1 & فصل زايش \\
\hline
\end{tabular}

صحيح نباشند. به طور كلى، سال زايش و سال تولد منابع

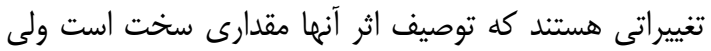

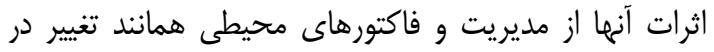

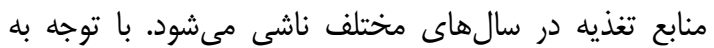

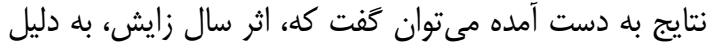

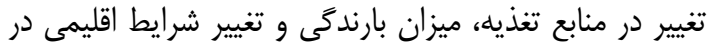

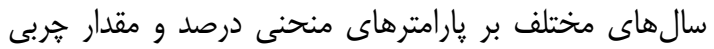
مؤثر است.

اثر دستجات سنى، هم كه حاصل تفريق سال تولد و سال

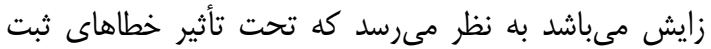

نتايج آناليز واريانس نشان داد كه، در هر دو نزاد و درد مورد

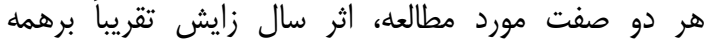

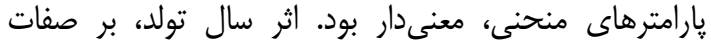

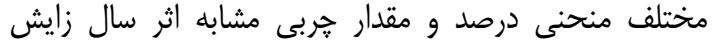

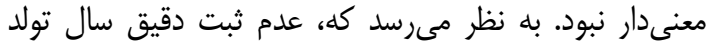

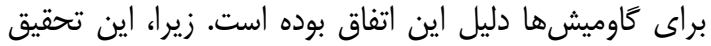

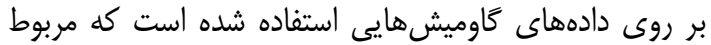

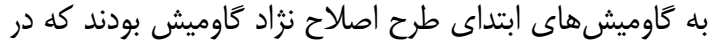

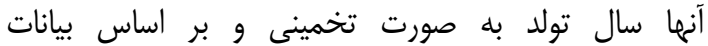

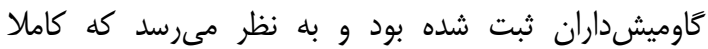




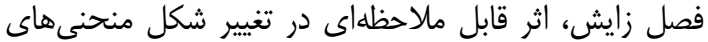

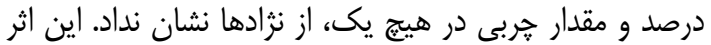

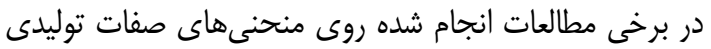

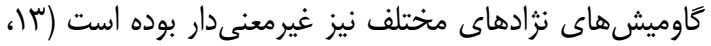

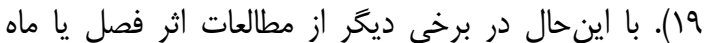
زايش، بر صفات منحنى توليد شير و و تركيبات آن معنى آنى داردار

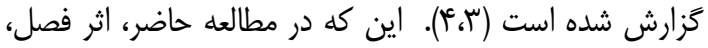

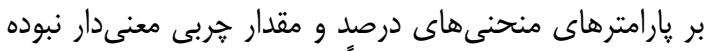

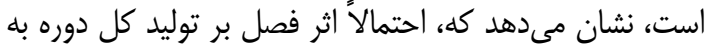

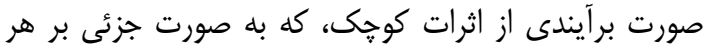

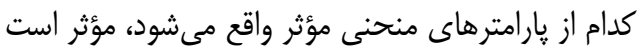

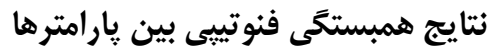

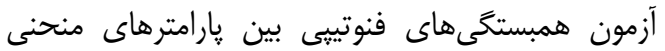

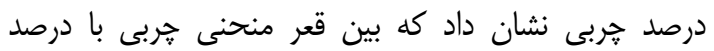

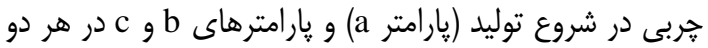

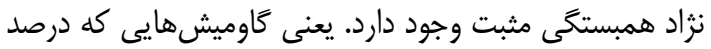

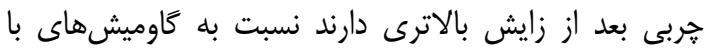

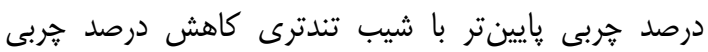

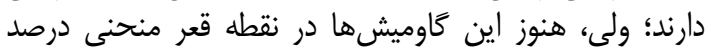

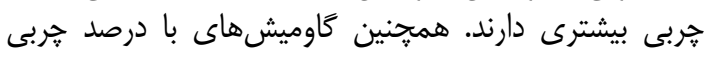

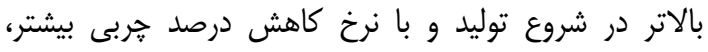

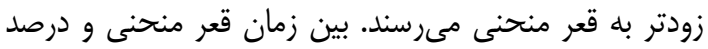

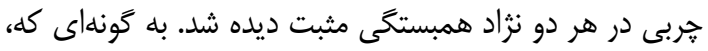

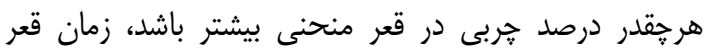

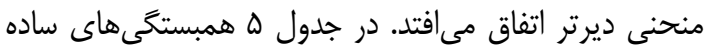

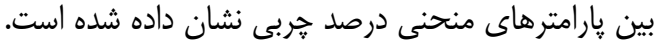

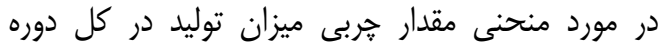

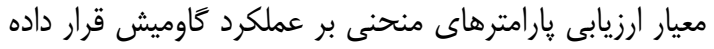

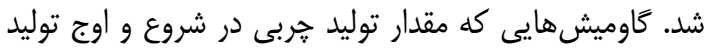

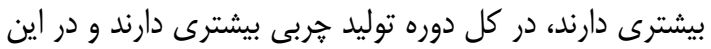

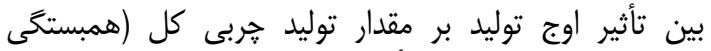
(Aه

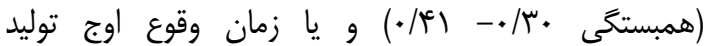

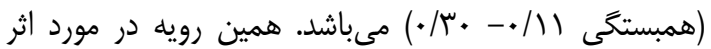

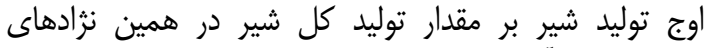

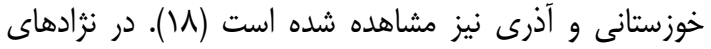

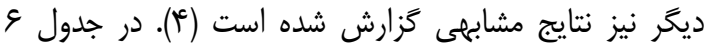

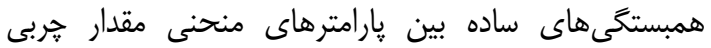

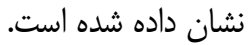

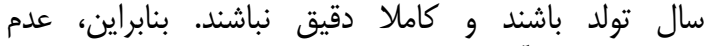

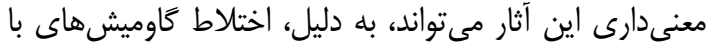

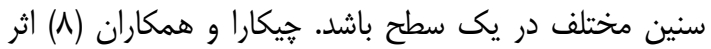

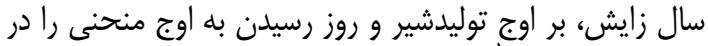

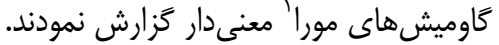

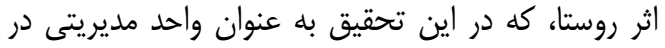

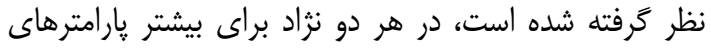

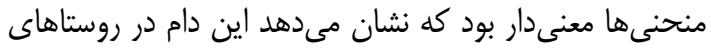

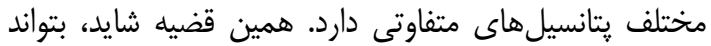

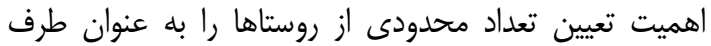

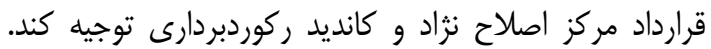

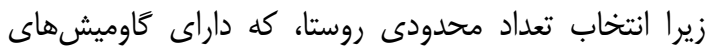

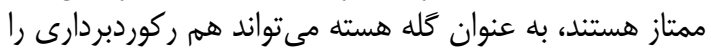

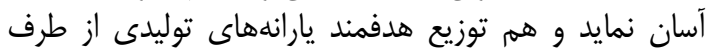

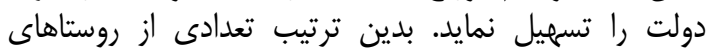

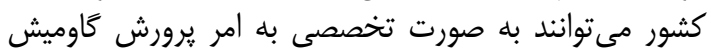

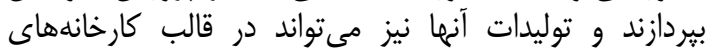

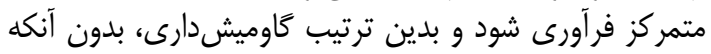

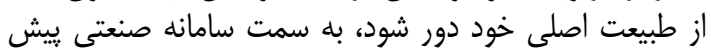

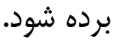
اثر دوره شيردهى، بر بيشتر :ارامترهاى منحنى درصد

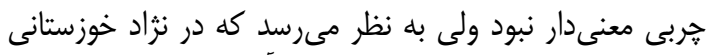

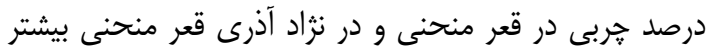

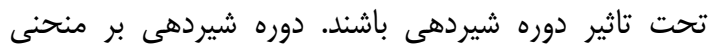

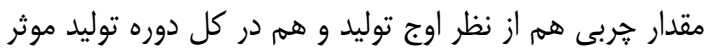

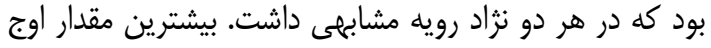

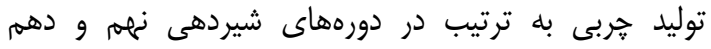

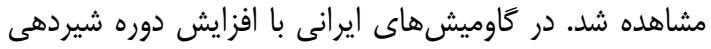

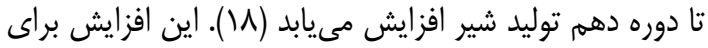

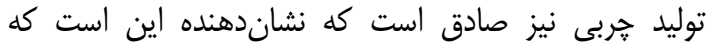

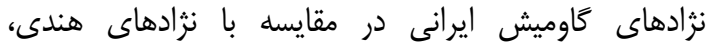

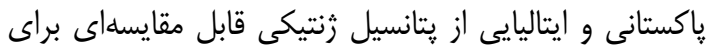

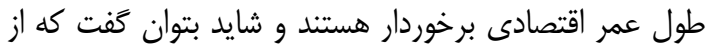

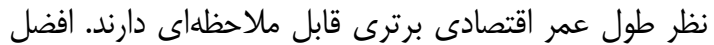

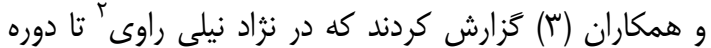

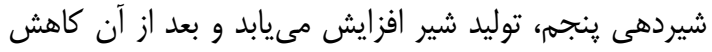

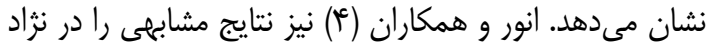

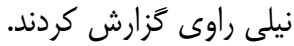


جدول ه- همبستخى بارامترهاى منحنى درصد حربى كاوميشهاى خوزستانى (بالاى قطر) و آذرى (بايين قطر) Table 5. Correlation coefficient of fat percentage curve parameter for Khuzestani buffalo (upper) and Azari (lower)

\begin{tabular}{|c|c|c|c|c|c|}
\hline $\mathrm{p}_{\mathrm{d}}$ & $p_{y}$ & $\mathrm{C}$ & $\mathrm{b}$ & $\mathrm{a}$ & \\
\hline.$- / \mathrm{Tr}^{\prime \prime}$ &.$/ 1 Q^{\prime \prime \prime}$ &.$/ 4 \omega^{*}$ &.$/ \mathrm{IV}$ & - & $\mathrm{a}$ \\
\hline$-\cdot / / V^{* \pi *}$ & $\cdot / \uparrow^{* N * *}$ & $\cdot / V e^{* \pi *}$ & - & $-\cdot / \mathrm{V}^{\pi * \pi}$ & $\mathrm{b}$ \\
\hline$\cdot / \Gamma \Lambda^{\pi * \pi}$ &.$/ 149^{* \pi * \pi}$ & - & $\cdot / N \mu^{\pi * \pi}$ &.$- / \mu r^{* \pi * \pi}$ & $\mathrm{c}$ \\
\hline$\cdot / \pi f^{\pi * \pi}$ & - & $\cdot|Q|^{\text {TN* }}$ &.$/ F \Delta^{\pi * \pi}$ & $\cdot \bigwedge^{\pi * \pi}$ & py \\
\hline - &.$|9|^{* \pi n}$ &.$/ \mu \mu^{* * * *}$ & $-\cdot / \pi e^{* \pi * \pi}$ & $\cdot|\Delta|^{* \cdots * \pi}$ & $\mathrm{pd}$ \\
\hline
\end{tabular}

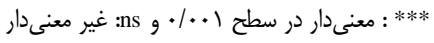

جدول צ- همبستخى پارامترهاى منحنى مقدار خربى گاوميشهاى خوزستانى (بالاى قطر) و آذرى (يايين قطر) Table 6. Correlation coefficient of fat percentage curve parameter for Khuzestani buffalo (upper) and Azari (lower)

\begin{tabular}{|c|c|c|c|c|c|c|}
\hline FatY & $\mathrm{p}_{\mathrm{d}}$ & $\mathrm{p}_{\mathrm{y}}$ & $\mathrm{c}$ & B & $\mathrm{a}$ & \\
\hline$\cdot|f|^{* * * *}$ &.$- / \mathrm{\Psi q}^{* * * *}$ & $\cdot / \Lambda^{* * * *}$ & $-\cdot / \Delta r^{* * * *}$ & $-.199^{* * *}$ & - & $\mathrm{a}$ \\
\hline$-\cdot / \Lambda^{* * *}$ & $\cdot / r \Lambda^{* * *}$ & $\cdot / \Lambda^{* * * *}$ & $\cdot / \Lambda \Delta^{* * *}$ & - & $-.199^{* * *}$ & b \\
\hline$-\cdot / r \Lambda^{* * *}$ &.$- / 11^{* * *}$ & $\cdot / 19^{* * *}$ & - & $\cdot / \Lambda r^{* * *}$ & $-\cdot / \uparrow \Lambda^{* * *}$ & $\mathrm{c}$ \\
\hline$\cdot / \Lambda \Delta^{* * *}$ & $-1 \cdot 9^{* * *}$ & - & $\cdot / \mu *^{* * *}$ & $\cdot / r \Delta^{* * * *}$ & $\cdot / 19^{* * *}$ & py \\
\hline$\cdot / 11^{* * *}$ & - & $-\cdot / 1 .^{* * *}$ & $\cdot / r \cdot{ }^{n s}$ & $\cdot / \mathbb{4} 8^{* * *}$ & $-\cdot / 9 r^{* * *}$ & $\mathrm{pd}$ \\
\hline - & $\cdot / \mu \cdot n$ & $\cdot / A \mathrm{~V}^{* * * *}$ & $-\cdot / M r^{* * *}$ & $-\cdot / r^{n}{ }^{n s}$ & $\cdot / \mu .^{* * * *}$ & $\mathrm{~m} 240$ \\
\hline
\end{tabular}

بررسى علل بروز آنها را ضرورى مىنمايد. عوامل

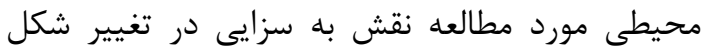

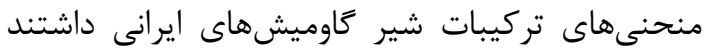

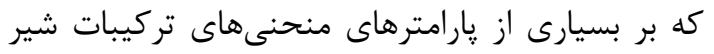

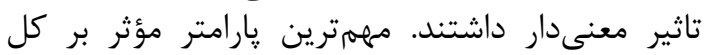

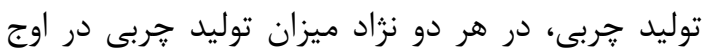

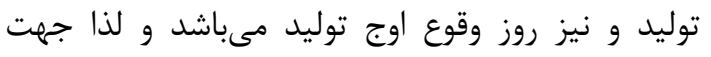
تغيير شكل منحنى مى توان روى اوج توليد توليد برنامه ريزى لون

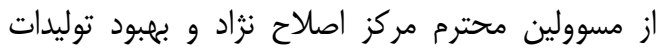

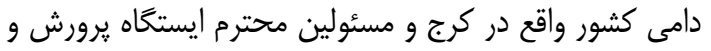

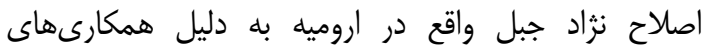

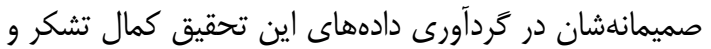

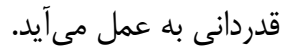

در نهايت مى توان خنين استنباط نمود كه، كاوميش باني

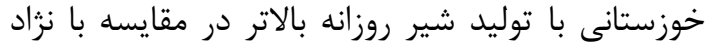

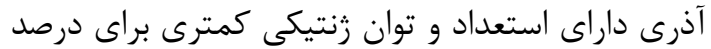

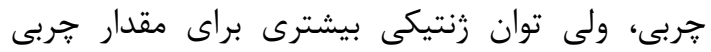

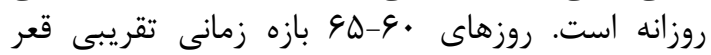

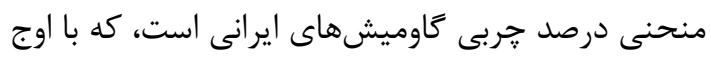

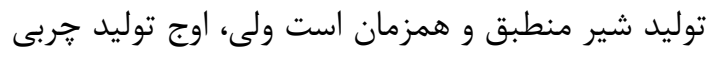

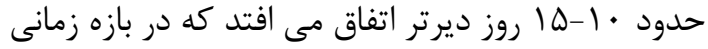

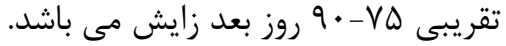

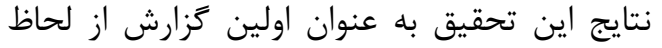

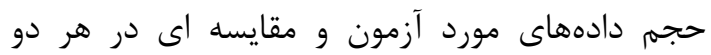

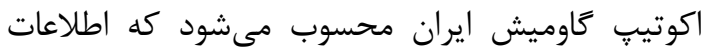

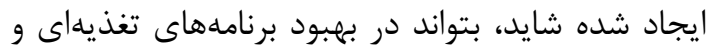

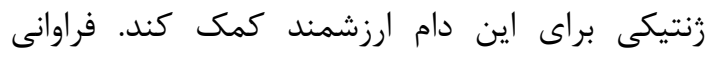

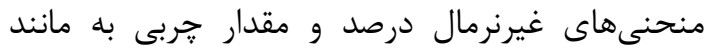

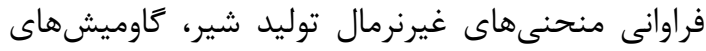

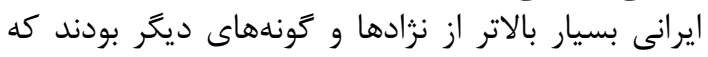


$\operatorname{lr}$. بررسى تأثير عوامل غيرزنتيكى بر مؤلفههاى منحنىهاى درصد ...

1. Abdel-Salam, S.A.M., W. Mekkawy, Y.M. Hafez, A.A. Zaki and S. Abou-Bakr. 2011. Fitting lactation curve of Egyptian buffalo using three different models. Egyptian Journal of Animal Production, 48(2): 119-133.

2. Adediran, S.A., A.E.O. Malau-Aduli, J.R. Roche and D.J. Donaghy. 2007. Using lactation curves as a tool for breeding nutrition and health management decisions in pasture based dairy systems, pp: 7478. Proceedings of the Dairy Research Foundation Symposium. The University of Sydney, Australia.

3. Afzal, M., M. Anwar and M.A. Mirza. 2007. Some factors affecting milk yield and lactation length in Nili Ravi buffaloes. Pakistan Veterinary Journal, 27: 113-117.

4. Anwar, M., P.J. Cain Rowlinson, P.M.S. Khan, A. Muhammad and E.M. Babar. 2009. Factors affecting the shape of the lactation curve in Nili-Ravi buffaloes in Pakistan. Pakistan Journal of Zoology, 9: 201-207.

5. Bahmani, B., A. Aslaminejad, M. Tahmorespour and K. Hasanpur. 2013. Study of milk yield and fat yield curves of some Iranian buffalo breeds. Journal of Animal Science (Pajohash va Sazandagi), 100: 36-44 (In Persian).

6. Barbosa, S.B.P., R.G.A. Pereira Santoro, K.R. Batista and R. Neto. 2007. Lactation curve of crossbred buffalo under two production systems in the Amazonian region of Brazil. Italian Journal of Animal Science. 6: 1075-1078.

7. Catillo, G., N.P. Macciotta, A. Carretta and A. Cappio-Borlino. 2002. Effects of age and calving season on lactation curves of milk production traits in Italian water buffaloes. Journal of Dairy Science, 85: 1298-1306.

8. Chhikara, S.K., N. Singh and S.S. Dhaka. 1998. Effect of some non-genetic factors on peak yield and days to attain peak yield in Murrah buffaloes. Proceedings of the $6^{\text {th }}$ World Congress on Genetics Applied to Livestock Production, pp: 481-484. Armidale, Australia.

9. Dijkstra, J., J. Franc, M.S. Dhanoa J.A. Maas M.D. Hanigan, A. Rook and D.E. Beever. 1997. A model to describe growth patterns of the mammary gland during pregnancy and lactation. Journal of Dairy Science, 80: 2340-2354.

10. Dimauro, C., G. Catillo, N.Bacciu and N.P.P. Macciotta 2005. Fit of different linear models to the lactation curve of Italian water buffalo. Italian Journal of Animal Science, 4: 22-24.

11. Hassanpur, K., M. Naghus, A. Aslami Nejad, B. Bahmani and M.A. Hashemi. 2012. Comparison of mathematical functions in the fitting of milk production curves and fatty production of Iranian buffaloes. The $5^{\text {th }}$ Iranian Congress of Animal Sciences. Isfahan University of Technology, (In Persian)

12. Naghus, M., K. Hassanpur, B. Bahmani, A. Aslaminejad and D. Kianzad. 2012. Assessment of Khuzestan and Azari buffalo production pattern under different environmental factors. The $5^{\text {th }}$ Iranian Congress of Animal Sciences. Isfahan University of Technology (In Persian).

13. Penchev. P., M. Boichev, Y. Ilieva and T.Z. Peeva 2011. Effect of different factors on lactation curve in buffalo cows. Slovak Journal of Animal Science, 44(3): 103-110.

14. Rahmaninia, J., H. Farhangfar, H.R. Mirzaee Emam Jomeh, N. Kashan and M.B. Sayadnejad. 2008. Phenotypic correlation of parameters and characteristics of lactation curve calculated by GammaWood function in Iranian buffaloes. Agricultural Science Journal, 31(2): 111-120 (In Persian).

15. Rahmaninia, J., H. Farhangfar, H.R. Mirzaee, N. Emam Jomeh Kashan and M.B. Sayadnejad. 2009. Influence of environmental factors affecting the shape of lactation curve in iranian buffalo ecotypes. Iranian Journal of Animal Science, 40(2): 59-68 (In Persian).

16. Şeahin, A., Z. Ulutaş, Y. Arda, A. Yüksel and G. Serdar. 2015. Lactation curve and persistency of anatolian buffaloes. Italian Journal of Animal Science, 14: 150-157.

17. Shokrollahi, B. and K. Hasanpur. 2014. Study of individual lactation patterns of Iranian dairy buffaloes. Journal of Agriculture and Rural Development in the Tropics and Subtropics, 115: 125133.

18. Shokrollahi, B. and K. Hasanpur. 2015. The identified factors influencing the occurrence of abnormal lactation curves in Iranian buffaloes. Indian Journal of Animal Science, 85: 870-874.

19. Taheri Dezfuli, B., M. Babaei and A. Kardooni. 2018. Fitting Milk Curve and Its Compounds for Khuzestani Buffaloes Using Five Different Functions. Research on Animal Production, 9(19): 113123 (In Persian).

20. Tekerli, M., M. Kucukkebabci, N.H. Akalin and S. Kocak. 2001. Effects of environmental factors on some milk production traits, persistency and calving interval of Anatolian buffaloes. Livestock Production Science, 68: 275-281.

21. Wood, P.D.P. 1967. Algebraic model of the lactation curve in cattle. Nature, 216: 164-165.

22. Yousef, Y., M. Gholizadeh and M. Madad. 2018. The use of a bivariate random regression model for genetic analysis of milk yield in iranian native buffalo. Research on Animal Production, 9(19): 102112 (In Persian). 


\title{
Investigation of Non-Genetics Factors Affecting Milk-Fat Content and Milk-Fat Percentage Curves' Parameters In Azeri and Khuzestani Buffalo Breeds
}

\author{
Karim Hasanpur ${ }^{1}$, Seyed Abbas Rafat ${ }^{2}$, Arash Javanmard ${ }^{3}$ and Davood Kianzad ${ }^{4}$ \\ 1- Assistant Professors, Department of Animal Science, Faculty of Agriculture, University of Tabriz, Tabriz, Iran \\ (Corresponding author: Karimhasanpur@tabrizu.ac.ir) \\ 2 and 3- Professor and Assistant Professors, Department of Animal Science, Faculty of Agriculture, University of \\ Tabriz, Tabriz, Iran \\ 3- M.Sc. Staff, National Breeding Center and improvement of animal production, Karaj, Iran \\ Received: June 28, 2019 \\ Accepted: November 9, 2019
}

\begin{abstract}
The study of milk production and composition curves in buffalo provides key pieces of information about genetic potentials and management-related strategy to both breeders and farmers. The objective of this study was to identify of the non-genetic factors of parameters for fitted Wood function on milk fat content (MFC) and milk fat percentage (MFP) curve of Azari and Khuzestan buffalo. The data were collected from 15065 and 15225 from Khozatani buffalo and 9571 and 9721 lactation period of Azari buffalo on MFC and MFP were used for further analyses. For the first phase, the Wood function for each individual within the investigated population was fitted. Then, environmental factors affected MFC and MFP in both populations were assessed. In the last step, after the estimation of curve parameters, the phenotypic correlation for both populations was estimated. Results of the present study showed the MFP of Khuzestan breed was almost \% 0.5 lower than Azari breed and faster reached to the lowest point within MFP curve shape. Furthermore, because of the higher milk production rate of Khuzestan breed, the MFC of this breed showed almost \% 25 higher at the peak, compared to the Azari buffalo. Also, due to higher observation of daily milk production in Khuzestan buffalo, this breed has good potential to produce more daily MFC and less MFP than Azari. Finally, the outputs of the report this research may be effective in help to improving nutritional the feeding formulation and genetics programs of Iranian buffaloes.
\end{abstract}

Keywords: Buffalo, Milk-Fat Curves, Wood Function, Production Peak, Correlation 